women) and kneeling for more than $2 \mathrm{~h}$ per day among men (OR 1.8 (1.0-3.0)).

Conclusions This study, conducted in a large representative working population, highlights the relation between incident knee pain and occupational risk factors such as handling loads and kneeling.

\section{PROBLEMS OF VISION, HEARING AND BALANCE AND RISKS OF WORKPLACE INJURY}

Keith Palmer, Stefania D'Angelo, E Clare Harris, Cathy Linaker, David Coggon. University of Southampton, MRC Lifecourse Epidemiology Unit, Southampton, UK

\subsection{6/oemed-2014-102362.20}

Objectives To assess the role of sensory impairments and disorders of balance in occupational injury.

Method The Clinical Practice Research Datalink records all medical consultations, referrals and diagnoses in primary care for $6 \%$ of the British population. Using this register we identified 1348 working-aged patients who had consulted medical services over a 20 -year period for workplace injury (cases) and 6652 matched controls. Risks were assessed in relation to visual impairment, common eye diseases, hearing loss, perforated ear drum, non-acute otitis media, and disorders of balance, using conditional logistic regression.

Results In all, 173 subjects had had an eye problem before the date of injury consultation (index date), 793 an ear problem (including 336 with impaired hearing and 482 with non-acute otitis media), and 266 a disorder of balance. No associations were found with specific eye diseases or perforation of the ear drum, but odds ratios (ORs) were moderately elevated for eye and ear problems more generally, and higher still in relation to blindness or partial sight (OR 1.90) and non-acute otitis media (OR 2.03). The OR for consulting with disorder of balance within the 12 months immediately preceding injury consultation was 1.81 (95\% CI 1.03-3.17).

Conclusions Problems of vision, impairments of hearing, and disorders of balance may all carry a moderately increased risk of occupational injury.

\section{OCCUPATIONAL EXPOSURE TO HAND-TRANSMITTED VIBRATION AND RISK OF DUPUYTREN'S CONTRACTURE}

${ }^{1}$ Keith T Palmer, 'Stefania D'Angelo, ${ }^{1}$ Holly Syddall, ${ }^{2}$ Michael I Griffin, ${ }^{1}$ Cyrus Cooper, 'David Coggon. 'University of Southampton, MRC Lifecourse Epidemiology Unit, Southampton, UK; '2University of Southampton, Institute of Sound and Vibration Research, Southampton, UK

\subsection{6/oemed-2014-102362.2}

Objectives To assess the relation between Dupuytren's contracture and occupational exposure to hand-transmitted vibration (HTV).

Method We mailed a questionnaire to 21201 subjects of working age, chosen randomly from the age-sex registers of 34 general practices in Great Britain and to 993 subjects randomly selected from military pay records. We asked about occupational exposure to 39 sources of HTV and about fixed flexion contracture of the little or ring finger. Analysis focused on men at work in the previous week, for whom estimates were made of average daily vibration dose $(A(8)$ r.m.s.). Associations with Dupuytren's contracture were estimated by Poisson regression, both for lifetime exposure to HTV and for exposures $>A(8)$ of $2.8 \mathrm{~ms}^{-2}$ r.m. s.in the past week, with adjustment for age, smoking status, social class and manual activities.

Results Full information on the study variables was available for 4969 eligible men, including 72 men with a history of Dupuytren's contracture, 2287 with occupational exposure to HTV, and 409 with $A(8)>2.8 \mathrm{~ms}^{-2}$ in the past week. RRs from occupational exposure were elevated 1.5 -fold, and were higher still for $A(8)>2.8 \mathrm{~ms}^{-2}$ (adjusted RR 2.85, (95\% CI 1.3-5.97).

Conclusions Our findings suggest that risk of Dupuytren's contracture is importantly elevated in men with high levels of weekly exposure to HTV.

\section{LOWER-EXTREMITY MUSCULOSKELETAL DISCOMFORT AND FATIGUE IN AUTOMOTIVE ASSEMBLY WORKERS}

${ }^{1}$ Jason Sarte, ${ }^{1}$ Stephanie Barnhart, ${ }^{2}$ Rebecca Moreland, ${ }^{1}$ Erin Moshier, ${ }^{1} J a m e s$ Godbold, 'Philip Landrigan. 'Icahn School of Medicine at Mount Sinai, New York, NY, USA; ${ }^{2}$ Scientific Advisory Committee, Detroit, Michigan, USA

\subsection{6/oemed-2014-102362.22}

Objectives To characterise the prevalence of musculoskeletal discomfort and fatigue of the lower extremities (LE) in a large sample of workers in a large, unionised automotive assembly facility. To assess whether floor matting reduced reported prevalence of LE discomfort.

Method Questionnaires were administered to 1353 workers representing 39\% of the plant's workforce. Discomfort of the LE was assessed on a qualitative scale. Participants rated tiredness overall and in the legs at the end of their shifts. Information on job designation, use of matting, history of LE or back injury, and use of over the counter pain medication was collected.

Results Highest mean discomfort was reported in the feet (3.04, $\mathrm{SD}=1.04)$ and lowest in the hips $(1.50, \mathrm{SD}=1.30)$. Assembly line or inspection/repair had the highest adjusted mean for discomfort for each body area. Gender- and age-adjusted fatigue ratings overall and in the legs differed significantly based on job designation $(p<0.0001)$. The percent of workers reporting matting, fall-related injuries and use of over the counter medication differed significantly by job designation ( $p<0.0001$, $\mathrm{p}<$ 0.0001 , and $\mathrm{p}=0.0447$, respectively). Among inspection/repair employees, there was no significant difference in mean discomfort and fatigue ratings between those who used matting and those who did not.

Conclusions There is a high prevalence of self-reported musculoskeletal discomfort and fatigue among automotive assembly workers. The difference in reported discomfort and fatigue levels by job designation warrants investigation into factors such as time spent standing and posture during work. Matting did not appear to reduce prevalence of LE discomfort.

\section{LONG-TERM NIGHTSHIFT WORK AND BREAST CANCER RISK IN HONG KONG WOMEN: RESULTS UPDATE}

'Lap Ah (Shelly) Tse, ${ }^{1}$ Feng Wang, ${ }^{2}$ Wing Cheong Chan, ${ }^{3}$ Çherry Wu, ${ }^{1}$ Mengjie Li, ${ }^{4}$ Chi Hei Kwok, ${ }^{6}$ Siu Lan Leung, Wai Cho Yu, Ignatius Tak-sun Yu. ${ }^{1} J C$ School of Public Health and Primary Care, The Chinese University of Hong Kong, Hong Kong, China; ${ }^{2}$ Breast Surgery NTEC Hospital Authority, North District Hospital, Hong Kong, China; ${ }^{3}$ Breast Pathology Service, North District Hospital and Alice Ho Miu Ling Nethersole Hospital, Hong Kong, China; ${ }^{4}$ Department of Oncology, Princess Margaret Hospital, Hong Kong, China; ${ }^{5}$ Department of Medicine and Geriatrics, Princess Margaret Hospital, Hong Kong, China; ${ }^{6}$ Department of Surgery, Yan Chai Hospital, Hong Kong, China

10.1136/oemed-2014-102362.23 